\title{
Strahlen im Kalten Krieg. Zusammenfassung und Schluss
}

Strahlen avancierten in der Schweiz erst nach 1945 zu einem Problem des Regierens. Fasziniert von möglichen Anwendungen von Strahlen in einem nuklearen Alltag und überzeugt von der Bewältigbarkeit eines künftigen Atomkrieges, begannen sich verschiedene Bundesstellen bereits kurz nach den Atombombenabwürfen auf Hiroshima und Nagasaki für Strahlen zu interessieren. In den folgenden Jahrzehnten war das Regieren von Strahlen von den Bedingungen und Konjunkturen des internationalen Kalten Krieges geprägt, aber auch von innenpolitischen Konflikten um die militärische und vor allem die zivile Nutzung der Atomenergie.

Der schweizerische Kalte Krieg zeichnete sich durch eine starke „Nuklearität“ aus, weil wichtige gesellschaftliche Auseinandersetzungen, maßgebende Verteidigungskonzepte und leitende Bedrohungsszenarien an die zunehmende Verfügbarkeit von Atomenergie und Strahlen gekoppelt waren. Das gouvernementale Handeln zielte darauf ab, die Zirkulation dieser Stoffe und Energiequellen zu ermöglichen. Dafür musste es im Gegenzug die Herstellung von Strahlensicherheit gewährleisten. Um dieses Ziel zu erreichen, wurde eine enorme Menge an Personal, Ressourcen, Geräten und Ideen in Sicherheitsdispositive gegen Strahlen eingebunden. Im Verlaufe des Kalten Krieges waren so unterschiedliche Akteure wie Wissenschaftler, Industrieunternehmen, Armeeoffiziere, Kernkraftwerkbetreiber, kantonale Laboratorien und Pflegefachleute, aber etwa auch die Schweizerische Unfallversicherungsanstalt, die Schweizerische Rundspruchgesellschaft und der Blutspendedienst des Schweizerischen Roten Kreuzes in die Produktion von Strahlensicherheit involviert: In dieser umfassenden gesamtgesellschaftlichen Mobilisierung spiegelt sich die politische Kultur des schweizerischen Kalten Krieges wider.

Im ersten Jahrzehnt des Kalten Krieges bestimmte die Vorbereitung auf einen künftigen Atomkrieg das gouvernementale Interesse an ionisierenden Strahlen und radioaktiven Stoffen. Dabei verfügten die Bundesbehörden zunächst nur über äußerst bescheidenes Wissen und Know-how. In der bereits 1945 gegründeten Schweizerischen Studienkommission für Atomenergie, aber auch in der im Zuge der Fallout-Debatte 1956 eingesetzten Eidgenössischen Kommission zur Überwachung der Radioaktivität gaben deshalb Wissenschaftler der verschiedenen Schweizer Hochschulen, namentlich Physiker 
und Mediziner, später auch Biologen und Chemiker, den Ton an. Innerhalb der Bundesverwaltung war es vor dem Hintergrund der angestrebten totalen Landesverteidigung zunächst hauptsächlich das Militär, das sich durch die Förderung der Strahlenforschung und den Aufbau eines ABC-Schutzdienstes Wissen über die gesundheitsschädigenden Wirkungen der Radioaktivität sowie über mögliche Strahlenschutzmaßnahmen aneignen wollte. Bei der dazu notwendigen Entwicklung von Geräten kam es zu mehreren gewinnbringenden Kooperationen zwischen dem Militär und der schweizerischen Industrie, so bei der Herstellung eines Teilchenbeschleunigers oder bei der Produktion von Strahlenmessgeräten. Gleichzeitig nutzten die Schweizer Forscher alle verfügbaren Kontakte, vor allem in den USA, aber auch in Großbritannien, der Bundesrepublik Deutschland und weiteren Ländern des ,Westblocks', um als notwendig erachtetes Know-how, Wissen und Material zu erlangen. Die, strategische Multioptionalität', mittels welcher die Schweiz versuchte, von vielfältigen transnationalen Wissenstransfers zu profitieren, kontrastierte mit den im Rahmen der totalen Landesverteidigung propagierten Autarkievorstellungen, welche die nationalen Joint Ventures prägten. Nationalen Alleingängen waren auch bei der Regulierung von Strahlen Grenzen gesetzt. Die Verpflichtung der Schweiz, bestimmte bindende Abkommen umzusetzen, aber auch das Erfordernis, die nationalen Strahlenschutzbestimmungen mit internationalen und später auch mit europäischen Normen kompatibel zu machen, führten dazu, dass die Formulierungen der 1963 in Kraft tretenden schweizerischen Strahlenschutzverordnung während des ganzen Kalten Krieges jeweils so weit möglich mit internationalen Bestimmungen harmonisiert wurden. Gleichzeitig waren es vielfach auch wirtschaftliche Interessen, welche die Ausgestaltung des Strahlenschutzes beeinflussten.

Ab dem Ende der 195oer bis zum Ende der 196oer Jahre wurde im Kontext des internationalen Kalten Krieges unter dem Slogan Atoms for Peace die zivile Nutzung der Atomenergie vorangetrieben. In der Folge verschob sich der Fokus der Strahlenforschung wie auch der Strahlenüberwachung immer mehr auf den expandierenden nuklearen Alltag. Die steigende Verfügbarkeit von Radioisotopen und hochenergetischen Strahlenquellen befeuerte nicht nur die Strahlenmedizin und hier insbesondere die Krebstherapie und -diagnostik, sondern auch die aufstrebende molekularbiologische Forschung. Die Forschungsförderung der $195^{8}$ geschaffenen, dem Schweizerischen Nationalfonds zur Förderung der wissenschaftlichen Forschung angegliederten Kommission für Atomwissenschaft wirkte in dieser Phase als bedeutender Katalysator für die biomedizinische Forschung in der Schweiz. Auch im Bereich der Strahlenüberwachung verlagerte sich das Gewicht der durchgeführten Messungen 
zunehmend auf die Abgabe von radioaktiven Stoffen durch Industriebetriebe, Spitäler und Atomanlagen.

Die 1970er Jahre erwiesen sich für das Regieren von Strahlen als wichtiges Jahrzehnt der Transformation. Wesentlich vorangetrieben durch die virulenten gesellschaftlichen Auseinandersetzungen um die zivile Nutzung der Atomenergie, rückten mögliche Kernkraftwerkunfälle und andere Katastrophenszenarien des nuklearen Alltags als neue Strahlenrisiken in die öffentlich-politische Diskussion. Dadurch weitete sich die Wahrnehmung der atomaren Gefahren auf den Zivilbereich aus und neuartige Bedrohungsvorstellungen wie eine mögliche Sabotage in Atomkraftwerken gewannen bei den schweizerischen Gesamtverteidigern stark an Plausibilität. Gleichzeitig rückte während der Entspannungsphase des Ost-West-Konfliktes das Bedrohungsbild eines Atomkrieges vorübergehend in den Hintergrund. Dieser Wandel in der wahrgenommenen Bedrohungslage spiegelte sich in den regelmäßig durchgespielten nationalen Verteidigungsübungen, aber auch in den im Zuge der Verwissenschaftlichung der Sicherheitspolitik neu erarbeiteten Bedrohungsstudien wider. In diesen unterschiedlichen Formen der Simulation, die darauf abzielten, das Gesamtverteidigungssystem zu testen und fortlaufend zu optimieren, wurde der "war-like character" des schweizerischen Kalten Krieges permanent aktualisiert. Über diese ständige performative Reifikation der imaginierten Bedrohungen wurden auch gesellschaftliche Ausgrenzungslinien gezogen - etwa dann, wenn der demokratische Protest gegen den Bau von Atomkraftwerken in den entworfenen Szenarien mit der subversiven Tätigkeit ,innerer Feinde‘ und der Gewaltbereitschaft ,linksterroristischer Gruppierungen assoziiert wurde. Die gouvernementale Sicht auf die AntiAtomkraft-Bewegung wurde folglich von den Bedrohungs- und Feindbildern des Kalten Krieges mitgeformt.

Das Szenario der Nuklearkatastrophe bildete sowohl im Militär- als auch im Zivilbereich die zentrale Bedrohung des Kalten Krieges. Bereits ab den 197oer Jahren war mit der Ausweitung und Verlagerung der Atomgefahr auf den Zivilbereich auch eine Neuausrichtung der schweizerischen Gesamtverteidigung verbunden. Die Konzeptionen für die Alarmorganisation für den Fall erhöhter Radioaktivität sowie für den Koordinierten Sanitätsdienst sollten deshalb einen flexiblen Übergang von Unfällen des nuklearen Alltags auf große atomare Katastrophen- und Kriegsereignisse und von den Bedingungen des Normalfalls auf die Vorbereitungen für den Notfall ermöglichen. In diesen hybriden Organisationen verdeutlicht sich die "totale Politik“ des schweizerischen Kalten Krieges: In ihnen vermischten sich Ziviles und Militärisches, womit auch die Grenzen zwischen Frieden und Krieg unscharf wurden. 
Durch die Anti-Atomkraft-Debatte geriet die gouvernemental propagierte zivile Nutzung der Atomenergie in den 1970er Jahren stark unter Druck. Damit begann ein wesentlicher Pfeiler des schweizerischen Kalte-KriegKonsenses brüchig zu werden. Gleichzeitig avancierten öffentlich-medial verbreitete Strahlenängste und zivilgesellschaftliche Kritik an der Kernenergie zunehmend zu einem nicht vernachlässigbaren politischen Faktor. Die maßgebenden Strahlenschutzakteure aus der Verwaltung, dem Militär, der Wissenschaft und der Wirtschaft waren deshalb bestrebt, gegenüber der erstarkenden Anti-Atomkraft-Bewegung in der schweizerischen Öffentlichkeit geeint aufzutreten. Obwohl mitunter langwierige Konflikte um Kompetenzen und Vormachtstellungen im Strahlenschutz ausbrachen, so etwa zwischen der Eidgenössischen Kommission zur Überwachung der Radioaktivität und den nuklearen Sicherheitsbehörden des Bundes, begünstigten die Auseinandersetzungen um den Bau von Kernkraftwerken insgesamt die Konsensbereitschaft innerhalb der "small world“ des schweizerischen Strahlenschutz-Netzwerkes. Der Reaktorunfall im US-amerikanischen Harrisburg von 1979 fügte der Unterstützung der Atomenergie auch in der Schweiz einen bedeutenden Dämpfer $\mathrm{zu}$ und trug Anfang der 1980er Jahre zum Entschluss bei, die Nutzung der Atomenergie und den Schutz vor Strahlen künftig in zwei separaten Gesetzen zu regeln.

Das Regieren von Strahlen begann sich folglich bereits in den 1970er Jahren zu wandeln. Der gravierende Kernkraftwerkunfall von Tschernobyl 1986 beschleunigte diese Veränderungen aber deutlich. Insbesondere verstärkte sich die Tendenz, den zivilen Bevölkerungsschutz im Rahmen der schweizerischen Gesamtverteidigung auf einen in Friedenszeiten stattfindenden, zivilen (atomaren) Notfall auszurichten. Um der öffentlich-medialen Kritik am Handling dieses Katastrophenereignisses durch die Behörden des Bundes und hier insbesondere die Alarmorganisation der Eidgenössischen Kommission für ACSchutz zu begegnen, fand eine Professionalisierung der Sicherheitsdispositive gegen Strahlen statt, die mit einer Aufwertung der zuständigen Verwaltungsstellen und einer Abwertung der auf Milizbasis arbeitenden Expertenkommissionen einherging. Damit verloren zahlreiche Akteure, namentlich die Eidgenössische Kommission für AC-Schutz, die Eidgenössische Kommission zur Überwachung der Radioaktivität, die Eidgenössische Kommission für die Sicherheit von Atomanlagen und die Eidgenössische Kommission für Strahlenschutz, die das Regieren von Strahlen in der Schweiz seit 1945 stark geprägt hatten, bedeutend an Einfluss.

Gleichzeitig büßten am Ende des Kalten Krieges auch Armee und Zivilschutz deutlich an gesellschaftlicher Zustimmung ein. Die erneute atomare 
Aufrüstung während des Zweiten Kalten Krieges zu Beginn der 198oer Jahre hatte in der Schweiz wie in vielen anderen Ländern in ,West' und ,Ost' ${ }^{\prime} \mathrm{zu}$ einem Wiedererstarken der Friedensbewegung sowie einer zunehmend lauter werdenden Kritik am Machbarkeitsglauben sowie an Sinn und Zweck von Zivilschutz und Armee geführt. Kritische Wissenschaftlerinnen und Wissenschaftler, aber auch breite Kreise der schweizerischen Bevölkerung stellten ein Weiterleben nach der Bombe infrage. Eine Studie der Zentralstelle für Gesamtverteidigung, die sich dieser Problematik widmete, musste am Ende des Jahrzehnts eingestehen, dass die absolut verheerenden sozialen, politischen und ökologischen Auswirkungen eines Atomkrieges bislang sträflich unterschätzt worden waren. Indem sich die Bewältigbarkeit eines Nuklearkrieges immer offensichtlicher als technokratische Illusion erwies, verloren die auf das Überleben des nationalen Kollektivs ausgerichteten Zivilschutzund Verteidigungsmaßnahmen entscheidend an gesellschaftlicher Unterstützung und Glaubwürdigkeit.

Die in diesem Buch erzählte Geschichte des Regierens von Strahlen verdeutlicht, wie die Dichotomien und Verläufe, Bedrohungsvorstellungen und Wahrnehmungen des Kalten Krieges einen wesentlichen Einfluss auf die Herstellung von Strahlensicherheit in der Schweiz ausübten. In dieser historischen Konstellation bildeten sich Überwachungs-, Regulierungs-, Alarmierungs- und Rettungsdispositive heraus, die sowohl auf den expandierenden nuklearen Alltag als auch einen künftigen Atomkrieg abzielten. Die dominante Regierungsweise unterschied sich je nachdem, ob ein Sicherheitsdispositiv unter ,normalen' oder unter Notfallbedingungen zu funktionieren hatte. Im Normalfall dominierte die ,Verteilung' von Sicherheit, indem eine Vielzahl der oben genannten Akteure, aber auch einige private Organisationen eigenständig für einen Teilbereich des Strahlenschutzes verantwortlich zeichneten. Demgegenüber stand im Notfall die ,Koordination' von Sicherheit im Zentrum. Bei einem so verheerenden Ereignis wie einer Atomkatastrophe bestand die einzige Strategie zur Bewältigung darin, alle verfügbaren militärischen und zivilen, staatlichen und privaten Institutionen und Mittel sämtlicher föderalen Ebenen bestmöglich aufeinander abgestimmt einzusetzen. Der helvetische Notfallföderalismus funktionierte indessen, wie zahlreiche nationale Verteidigungsübungen stets von Neuem vor Augen führten, mehr schlecht als recht. Nicht zuletzt aufgrund immanenter Strukturprobleme des schweizerischen Gesamtverteidigungssystems blieb die ,koordinierte Sicherheit' während des ganzen Kalten Krieges prekär.

Die gegen Strahlen entworfenen Sicherheitsdispositive weiteten sich im Untersuchungszeitraum dieser Arbeit fortlaufend aus. So wuchsen nicht nur die zuständigen Verwaltungseinheiten und Expertenkommissionen, etwa die 
Sektion für Strahlenschutz oder die Abteilung für die Sicherheit der Kernanlagen, die Alarmorganisation für den Fall erhöhter Radioaktivität oder der Koordinierte Sanitätsdienst. Vielmehr nahmen auch die überwachten Personen und Gegenstände sowie die regulierten Anwendungen von Strahlen zu. Diese expansive Logik ist der Tendenz von Sicherheitsdispositiven geschuldet, fortlaufend neue Objekte zu integrieren. Studiert man - wie dies für die vorliegende Arbeit notwendig war - im Schweizerischen Bundes- und weiteren Archiven Quellenbestände zum Regieren von Strahlen im Kalten Krieg, so finden sich überwältigende Mengen an Protokollen, Konzepten, Abhandlungen, Briefen, Notizen, Dokumentationen, Berichten, Organigrammen, Plänen, Tabellen, Listen und Karten - und es ist anzunehmen, dass sich in diversen Staats-, Stadt-, Gemeinde- und Organisationsarchiven unzählige weitere Bestände zum Problem des Strahlenschutzes sichten ließen. Diese monströsen Papierberge sind ein Indikator für die große Bedeutung, welche dem Regieren von Strahlen in der Schweiz nach 1945 zukam. Die zunehmende Komplexität, welche den Umgang mit Strahlen im Verlaufe des Kalten Krieges kennzeichnete, verlangte nach dem Reaktorunfall von Tschernobyl mit Nachdruck nach professionellen Strukturen im Strahlenschutz.

Diese Ausdehnung der Sicherheitsdispositive gegen Strahlen lässt sich als Teil einer zunehmenden funktionalen Differenzierung der Bundesverwaltung beschreiben. Der Bund übernahm in der Nachkriegszeit eine Vielzahl neuer Aufgaben - so auch den Strahlenschutz -, die zu einem stetigen Ausbau seiner Verwaltung führten. Verwaltungsstellen avancierten damit zu zunehmend gestaltenden und steuernden gouvernementalen Strahlenschutzakteuren. Die schier endlosen Laufmeter archivierter Quellen verweisen jedoch auch auf den beispiellosen akribischen Eifer und die bemerkenswerte bürokratische Sorgfalt, mit welcher unzählige Personen und Institutionen über mehrere Jahrzehnte die Vorbereitung auf eine imaginierte Atomkatastrophe in Angriff nahmen. Nicht alle Konzeptionen wurden umgesetzt, nicht sämtliche Maßnahmen verwirklicht, nicht jedes Projekt abgeschlossen. Vieles wurde zu Makulatur - doch alles hinterließ Papier. In diesem papiernen Erbe manifestiert sich der Anspruch der permanenten preparedness der totalen Landesbzw. Gesamtverteidigung. Die politische Kultur des schweizerischen Kalten Krieges materialisiert sich so nicht nur, aber auch als ,Papierkrieg', der jedoch wie diese Arbeit gezeigt hat - vielfältige und nachhaltige ,reale' Effekte zeitigte.

Von den Plänen zur Beschaffung einer eigenen Atombombe über die erhoffte "Isotopenökonomie“ bis hin zu den Visionen unendlicher Energie durch Atomkraftwerke: Immer war den Sicherheitsdispositiven gegen Strahlen ein Zukunftsversprechen eingeschrieben gewesen: das Versprechen, Strahlensicherheit in jedem Fall herstellen zu können, sodass radioaktive 
Strahlung und Atomenergie gefahrlos und produktiv zirkulieren konnten. Diese positiv konnotierte Verschränkung von Atomenergie und Strahlenschutz hatte ermöglicht, dass Atomlobbyisten mit Strahlenbiologen in denselben Kommissionen Einsitz nahmen, Biowissenschaftler und Kernphysiker von denselben Forschungsförderungsinstitutionen unterstützt wurden und sich Militärstrategen und Spitaldirektoren an denselben Verteidigungsplanungen beteiligten. Was jahrzehntelang zusammen gedacht und geplant, aufgebaut und konzipiert worden war, begann in den 1970er Jahren durch die Kritik am Bau von Atomkraftwerken zu bröckeln und wurde nach dem Reaktorunfall Tschernobyl unwiderruflich auseinanderdividiert. Mit dieser einsetzenden strukturellen Loslösung war auch eine zunehmende mentale Entkopplung verbunden: die Preisgabe des gouvernementalen Konsenses zur uneingeschränkten Förderung von Atomenergie und radioaktiver Strahlung, das Eingeständnis einer Desillusionierung, ja einer Entmystifizierung dieser Energiequellen und Stoffe. Am Ende des Kalten Krieges hatten Strahlen ihre Erhabenheit verloren. 